\title{
A BRAIN COMPUTER INTERFACE TO CONTROL HOME APPLIANCES FOR EPILEPSY PATIENTS
}

\author{
Dr. R. mariappan ${ }^{1 *}$, M. G. Aarthi ${ }^{2}$, P. Kalaiselvi ${ }^{3}$, M. Kanimozhi ${ }^{4}$, \\ *1Professor, ${ }^{2,3,4}$ Students, \\ *1,2,3,4 Department of Information Technology Velammal Institute of Technology, Panchetti, Tamilnadu, India
}

*Corresponding Author: -

\begin{abstract}
: -
A brain computer interface (BCI) is a new communication channel between the human brain and the smart home appliances. EEG based non-invasive BCI with frequency filter algorithm was connected with a Virtual Reality system in order to control home appliances by using GUI platform. It offers an alternative to natural communication and control with human brain assumption and the ON OFF condition of home appliance is based on the muscle movement with interrupts.
\end{abstract}

Keywords: - Brain computer interface (BCI), Electroencephalography (EEG), Frequency filter algorithm, Graphical user interface (GUI).

\section{(a) (\$) (1)}




\section{INTRODUCTION}

A brain-computer interface (BCI), sometimes called a mind-machine interface (MMI) or brain-machine interface (BMI) is a direct communication pathway between the brain and an external device. BCIs are often directed at assisting, augmenting, or repairing human cognitive or sensory-motor functions. BCI records the different stages of EEG signals from the brain according to the intent of the user was connected with a Virtual Reality system in order to control a smart home appliances. It offers an alternative to natural communication and control. It is an artificial system that bypasses the body's normal efficient pathways, which are the neuromuscular output channels. Different brain states are the result of different patterns of neural interaction. These patterns lead to waves characterized by different amplitudes and frequencies. This neural interaction is done with multiple neurons. Every interaction between neurons creates a minuscule electrical discharge.This project dealing with signals from the brain. The signal generated by brain was received by the brain sensor and it will divide into packets and the packet data transmitted to wireless medium (blue tooth), the wave measuring unit will receive the brain wave raw data and it will convert into signal using MATLAB with GUI platform. Then the instructions will be sending to the home section to operate the modules (bulb, fan). This project is based on the thought process and the on off condition of home appliance is by changing the muscle movement with interrupts.

\section{II.BACKGROUND AND RELATED WORKS}

In this section, we explain the background and related works by various authors and their implementation methods for solving the problem in the BCI using different approaches and techniques.

Wei Tuck Lee, Humaira Nisar, Aamir S. Malik, Kim Ho Yeap ${ }^{[1]}$ proposed a method using a non-invasive BCI device known as Emotiv EPOC headset to record the brain signals. The transmitted EEG signals are interfaced with the computer via Bluetooth. The control of mouse cursor in the Emo control panel is driven by the built in gyro sensor in the Emotiv headset. The mouse click is performed by raising an eye brow.

K. Amarasinghe, D.Wijayasekara, M.Manic ${ }^{[2]}$ proposed a method for recognizing thought pattern for "move forward" and "rest" based on Self Organizing Maps (SOM). The presented thought recognition methodology is a three step process which utilizes SOM for unsupervised clustering of pre-processed EEG data and feed-forward Artificial Neural Networks (ANN) for classification. Any slight change in SOM map left the entire process ineffective.

Chin-Teng Lin, Bor-Shyh Lin, Fu-Chang Lin, Che-Jui Chang ${ }^{[3]}$ proposed a system consisting of wireless physiological signal acquisition module, an embedded signal processing module, a ctrl power line communication environmental controller, and a host system using UPnP. The physiological signal acquisition module and embedded signal processing module were designed for long-term EEG monitoring and backend analysis. Instant switching of UPnP is considerably difficult.

Vaibhav Gandhi, Girijesh Prasad, Damien Coyle, Laxmidhar Behera, Thomas Martin McGinnity ${ }^{[4]}$ proposed a robotic control mechanism by intelligent adaptive user interface (iAUI) based on an adaptive shared control with multiple movements. Epilepsy patients cannot perform multiple brain task to control a robot.

\section{METHODOLOGY}

The various steps explained here are as follows:

A. EEG signal acquisition

B. Frequency filter algorithm

C. Setup of virtual reality system

\section{A.EEG signal acquisition}

EEG signal is acquisted from brainwave sensing head band wirelessly connected to the computer. This head band consists of single dry electrode (channel) with three contacts: EEG; REF; and GND which is powered by three AAA batteries. It is mounted by placing the EEG electrode in the left or right eye on the forehead Fig 1: brainwave sensing headband. The feature extraction is done by brainwave visualizer for detecting the attention and meditation level of the person.

The raw EEG data output is 512 bits per second. Fig 2: brainwave visualizer measuring attention level.The EEG powerspectra includes delta, theta, alpha, beta, mu and lamda. The waves and their frequencies are given in the Fig 3: Frequency range of EEG power spectra

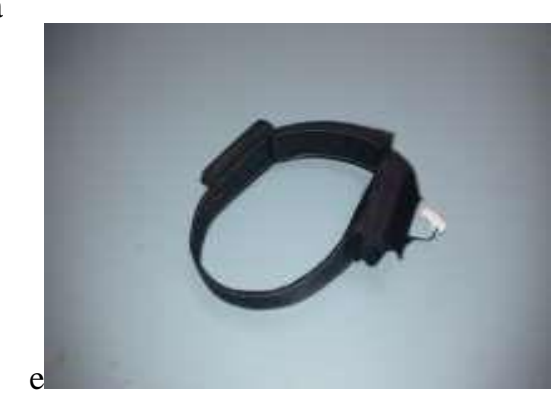

Fig 1: Brainwave sensing headband 


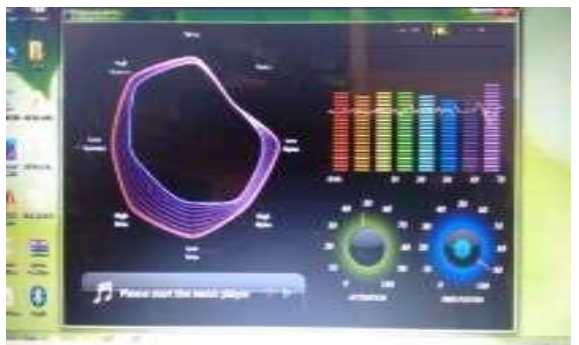

Fig 2: Brainwave visualizer measuring attention level

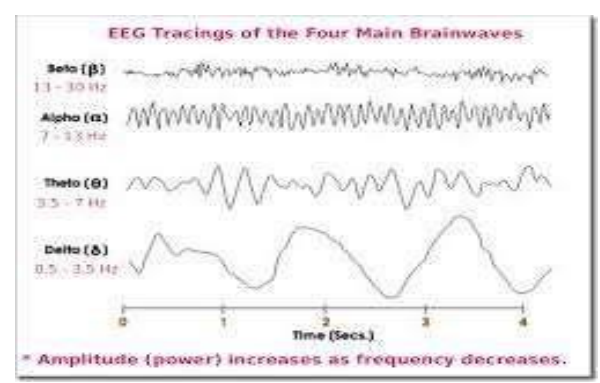

Fig 3: Frequency range of EEG powerspectra

\section{B. Frequency filter algorithm}

The signal to noise ratio (SNR) can be computed as,

$$
\operatorname{SNR}=\frac{\operatorname{mean}(\mu)}{\text { Standard deviation }(\sigma)}
$$

A signal mostly contains low frequency information. Hence the noise is dominant for high frequencies and its effects can be reduced using some kind of frequency filter. Frequency filtering is the method to remove unwanted frequency range (i.e). above cut off frequency from the recorded signal. Here we are using FIR symmetric filter.

\section{Algorithm:}

Let an FIR filter of length $\mathrm{M}$ has a frequency response

Where,

$$
\mathbf{H}(\mathbf{w})=\sum_{n=0}^{M-1}\left(b_{n} \mathbf{e}^{-\mathbf{j w n}}\right)
$$

$b_{n}$ is the filter co-efficient and also the unit impulse response of filter.

$$
\mathbf{h}(\mathbf{n})=\left\{\begin{array}{c}
b n, 0 \leq n \leq M-1 \\
0, \text { otherwise }
\end{array}\right.
$$

The linear phase condition is obtained by imposing symmetry condition on the unit impulse response of the filter. Let symmetry condition as

Case 1: $M=$ Odd

$$
\mathbf{h}(\mathbf{n})=\mathbf{h}(\mathbf{M - 1}-\mathbf{n})
$$

'The frequency response of linear phase FIR filter with symmetry response for M- odd is,

Case 2: $M=$ Even

$$
\mathrm{H}_{\mathrm{r}}(\mathrm{W})=h\left(\frac{M-1}{2}\right)+2 \sum_{n=0}^{(M-3) / 2}\left(\mathrm{~h}(\mathrm{n}) \cos \mathrm{w}\left(\left(\frac{M-1}{2}\right)-n\right)\right)
$$

'The frequency response of linear phase FIR filter with symmetry response for M- even is,

$$
\mathrm{H}_{\mathrm{r}(\mathrm{W})}=2 \sum_{n=0}^{(M / 2)-1}\left(\mathrm{~h}(\mathrm{n}) \cos \mathrm{w}\left(\left(\frac{M-1}{2}\right)-n\right)\right)
$$

\section{Setup of virtual reality system}

The brainwave sensing headband is then connected to the Virtual Reality System which is the ARM7 microcontroller. The $5 \mathrm{~V}$ operating voltage is supplied to the microcontroller through carpacitors, resistors, regulators and a crystal oscillator.The serial data gets inputed into the microcontroller circuit and with the help of the relay switches it gives control over the home appliances such as bulb,fan,etc,. The functional block diagram of the BCI system is shown below in Fig 4: Block diagram of BCI system. 


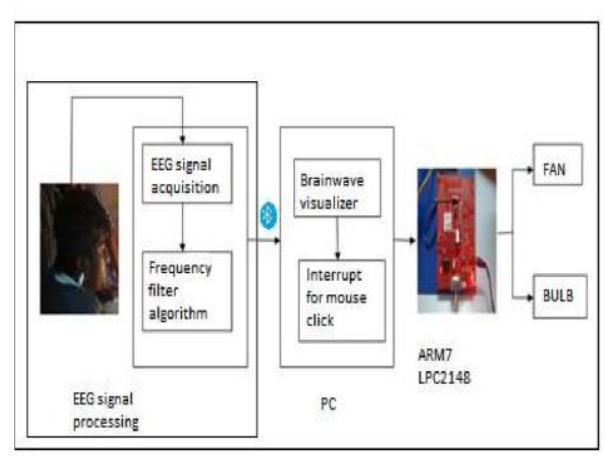

Fig 4: Block diagram of BCI system

\section{IV.OPERATION}

The brainwave sensing headband is used by the epilepsy patients or elderly people by the way the EEG signals from the brain are measured by the signal acquisition unit. The Frequency filter algorithm filters the noise from the signals and extracts the desired beta waves for use. The signals are sent to the PC via Bluetooth interface in the form of data packets. Brainwave visualizer reads the signal and shows the attention level of the user so that we can be able to fix their attention level for control in the MATLAB. The serial data is sent to the ARM7 LPC2148 microcontroller which then performs ON OFF operations of bulb, fan, etc, by mouse click in the GUI by the human thought process.

\section{V.CONCLUSION}

Here the proposed BCI for epilepsy patients will enable them to control the devices around them without the help of others. The noise is filtered to get the desired frequency which increases the efficiency of the BCI to $88 \%$. The system is easy to use and does not need assistance to mount it. For checking efficiency in various users we made an analysis and shown below in table

1: efficiency level check. We can also make further advancements in this system using temperature sensor and give fire alarm in case of emergency.

\begin{tabular}{|l|l|l|}
\hline USER & $\begin{array}{l}\text { ATTENTION } \\
\text { LEVEL }\end{array}$ & EFFICIENCY \\
\hline & & \\
\hline Ajith & 45 & $87 \%$ \\
\hline Aarthi & 65 & $85 \%$ \\
\hline Kani & 80 & $90 \%$ \\
\hline Kalai & 75 & $89 \%$ \\
\hline
\end{tabular}

\section{REFERENCES}

[1].Chin-Teng Lin, Fellow, IEEE, BorShyh Lin, Member, IEEE, Fu-Chang Lin, and Che-Jui Chang "Brain Computer Interface-Based Smart Living Environmental Auto-Adjustment Control System in UPnP Home Networking” in IEEE system journal 2014.

[2].Kamarasinghe,D.Wijayasekara,M.Mani c "EEG based brain activity monitoring using artificial neural networks" in University of Idhao,2014

[3].Vaibhav Gandhi, Girijesh Prasad, Damien Coyle,Laxmidhar Behera, and Thomas Martin McGinnity" EEG-Based Mobile Robot Control Through an Adaptive Brain-Robot Interface" in IEEE 2014

[4].Wei Tuck Lee, *1Humaira Nisar, *2Aamir S. Malik, 1**Kim Ho Yeap,Senior **Member IEEE” A Brain Computer Interface for Smart Home Control” University Tunku Abdul Rahman, 2 in IEEE internal symposium on consumer electronics(ISCE),2013,University Technology PETRONAS, Perak, Malaysia 